\title{
Acidemia orgánica (propiónica) en un neonato detectada por espectrometría de masas en tándem
}

\author{
Yolanda Cifuentes ${ }^{1}$, Isabel De la Hoz¹, Martha Bermúdez², Clara Arteaga ${ }^{1}$ \\ 1 Facultad de Medicina, Universidad Nacional de Colombia, Bogotá, D.C., Colombia \\ 2 Instituto de Genética Humana, Universidad Nacional de Colombia, Bogotá, D.C., Colombia \\ Institución donde se llevó a cabo el trabajo: Instituto Materno-Infantil, Universidad Nacional de \\ Colombia, Bogotá, D.C., Colombia
}

La acidemia propiónica es un error innato del metabolismo de herencia autosómica recesiva, causada por la deficiencia de la enzima propionil CoA carboxilasa, que cataliza la conversión de propionil CoA a malonil CoA. Es la principal causa de hiperglicinemia cetósica. En el período neonatal se manifiesta comúnmente con vómito, dificultad en la alimentación, hipotonía, letargia, acidosis metabólica, cetosis e hiperamonemia.

Se presenta el caso de una recién nacida con cuadro clínico de encefalopatía asociada a hipoglucemia, cetonuria, hiperamonemia, acidosis metabólica con anión gap aumentado, anemia, leucopenia y trombocitopenia en quien se diagnosticó una acidemia orgánica mediante espectrometría de masas en tándem.

Existían antecedentes de consanguinidad entre los padres, de un aborto, un mortinato y tres muertes neonatales, sugestivo de enfermedad genética.

Palabras clave: recién nacido, encefalopatías, cuerpos cetónicos, hiperamonemia, espectrometría de masas en tándem.

Neonatal onset of organic acidemia (propionic) diagnosed by tandem mass spectrometry

Propionic acidemia is an autosomal recessive disorder as a result of a deficient activity of propionyl-CoA carboxylase. Propionyl CoA is metabolized by propionyl-CoA carboxylase to methylmalonyl CoA. Propionic acidemia is a major cause of ketotic hyperglycinemia. This disorder is characterized by episodic vomiting, dehydratation, feeding intolerante, lethargy, hypotonia, metabolic acidosis, ketosis and hyperammonemia. The patient presented herein was a full-term female newborn with encephalopathy in the first days of life. She presented hypoglycemia, metabolic acidosis with increased anion gap, ketosis, hyperammonemia, anemia, leukopenia and thrombocytopenia. The brain ultrasonography was normal. The tandem mass expectrometry done by Pediatrix was abnormal, with the acylcarnitine results consistent with an organic acidemia. The parents are consanguineus and have a history of abortus, miscarriage and neonatal death, characteristics suggestive of the presence of genetic defects.

Key words: newborn, brain diseases, ketone bodies, hyperammonemia, tandem mass spectrometry.

La acidemia propiónica (MIM 232000 y 232050), la más común de las acidemias orgánicas, es un error congénito del metabolismo, de herencia autosómica recesiva, causada por la deficiencia

Correspondencia:

Yolanda Cifuentes, Calle 146 № 7a-74, Bogotá, D.C., Colombia Teléfonos: (315) 3335950 y (310) 6975064 mycifuentesd@unal.edu.co

Recibido: 26/04/07; aceptado: 14/11/07 de la enzima propionil CoA carboxilasa, que cataliza la conversión de propionil CoA a malonil CoA. La propionil CoA carboxilasa es una enzima mitocondrial intrahepática que tiene dos subunidades: la alfa, dependiente de biotina, cuyo locus está en el cromosoma 13 (13q32) y la beta, cuyo locus se halla en el cromosoma 3 (3q21-22). La deficiencia enzimática puede ser secundaria a una mutación para la subunidad alfa (PCCA), para la beta (PCCB) o para ambas (1-3). El término 
acidemia orgánica, o aciduria orgánica, se aplica a los trastornos caracterizados por la excreción en orina de ácidos orgánicos que resultan de la alteración en el catabolismo de los aminoácidos de cadena ramificada o de la lisina, debida a deficiencia de actividad enzimática (4).

Esta acidemia fue descrita por primera vez en 1961 por Child et al. (3). La frecuencia de la enfermedad puede ser tan alta como un caso por 18.000 habitantes. La prevalencia informada en los Estados Unidos es de un caso por cada 35.000 a 75.000 habitantes, pero podría ser mayor puesto que muchas muertes neonatales pueden deberse a acidopatías sin diagnosticar (2). Se encuentra una incidencia aumentada en Groenlandia (5) y, también, en Arabia Saudita a causa de los matrimonios entre parientes (6).

El ácido propiónico y el metabolito conocido como propionil coenzima $A(\mathrm{Co} A)$ son productos del catabolismo de aminoácidos de cadena ramificada (isoleucina, valina, metionina y treonina). El colesterol, los ácidos grasos raros y las bacterias intestinales también contribuyen a la producción de ácido propiónico. La deficiencia de la enzima produce acumulación de ácido propiónico que, al no poderse metabolizar, es hidrolizado; el propionato se une con la carnitina y forma el éster propionil-carnitina y, de esta manera, sale de la mitocondria y se hidroliza en sangre como propionato y carnitina; no obstante, debido a su gran volatilidad no es un metabolito útil para el diagnóstico, y son el metilcitrato y el 3hidroxipropionato los metabolitos que sirven para hacer el diagnóstico (1,7-9).

El defecto enzimático produce varios metabolitos potencialmente tóxicos que -se ha sugeridopueden ocasionar infartos en los ganglios basales por daño en las células endoteliales. Se ha comprobado que estas lesiones no se deben a hipoxia puesto que el hipocampo que es más sensible a ella, se encuentra indemne. Las hipótesis alternas implican disfunción directa de la citocromo-c-oxidasa o acumulación de glutamato en los astrocitos debida a la hiperamonemia frecuentemente presente en los cuadros de acidemia propiónica (2).

Este trastorno se caracteriza por una acentuada elevación de la glicina en plasma y orina por lo que inicialmente se le conoció como hiperglicinemia cetósica; sin embargo, otros trastornos metabólicos pueden cursar con hiperglicinemia (3).

La hiperglicinemia puede ser debida a un defecto primario en el sistema de escisión de la glicina o a bloqueo enzimático por metabolitos tóxicos, como sucede en las acidurias orgánicas o por drogas específicas como el valproato de sodio (10).

La acidemia propiónica en el período neonatal se manifiesta comúnmente con vómito, dificultad en la alimentación, hipotonía, letargia, acidosis metabólica, cetosis e hiperamonemia (11).

La hiperamonemia puede ser grave (12), el mecanismo por el cual se produce se desconoce y parece ser diferente al mecanismo que produce la hiperamonemia en los pacientes con defectos del ciclo de la urea (13).

En $80 \%$ de los casos la enfermedad se manifiesta en los primeros días de vida pero también ha sido descrita en lactantes, niños mayores y adultos (14). El compromiso cardíaco ha sido descrito en las formas de presentación tardía $(15,16)$.

En los pacientes con diagnóstico de acidemia propiónica, la presencia de movimientos coreoatetoides frecuentemente se debe a infartos de los ganglios basales (2). La atrofia del nervio óptico ha sido descrita en hombres con acidemia propiónica, independiente del control metabólico de la enfermedad (17).

Las manifestaciones clínicas pueden ser $(1,4,18$ 21):

- neurológicas: letargia, hipotonía, arreflexia o convulsiones;

- gastrointestinales: deshidratación importante por vómito, rechazo a la vía oral; la hepatomegalia es poco frecuente en el período neonatal;

- metabólicas: hipoglucemia, acidosis severa o cetosis;

- hematológicas: pancitopenia con predominio de neutropenia y trombocitopenia por metabolitos tóxicos que suprimen la función medular, lo que predispone a las infecciones; $\mathrm{y}$ 
- respiratorias: hiperventilación secundaria a la acidosis o al compromiso neurológico y quejido.

El curso clínico se caracteriza por episodios recurrentes de cetoacidosis precipitados por infecciones, ingestión excesiva de proteínas, ayuno y en los pacientes de mayor edad, por el ejercicio. En un estudio de 25 pacientes en Arabia Saudita se mostró que 14 pacientes presentaron un cuadro de acidosis, hiperamonemia y trombocitopenia mientras que en 11 la presentación fue inusual: dos recién nacidos tenían hiperamonemia, en dos el hallazgo fundamental fue el vómito, tanto que se les sospechó obstrucción intestinal, tres pacientes presentaron un trastorno inmunitario y cuatro tenían encefalopatía aguda o crónica (20).

El $80 \%$ de los pacientes de un estudio de 38 pacientes en Arabia Saudita, presentaba infección por gérmenes inusuales lo que sugiere inmunodeficiencia causada por un efecto de la enfermedad sobre los linfocitos T y B y sobre la medula ósea. Estas infecciones ocurrían a pesar de que los pacientes estaban manejados con dieta apropiada, carnitina y metronidazol durante las crisis (6).

En los lactantes hay retardo del desarrollo psicomotor, anormalidades en el electroencefalograma y osteoporosis. Las manifestaciones a largo plazo son de predominio neurológico, como retardo mental, demencia y corea.

La degeneración espongiforme de la sustancia blanca es un hallazgo frecuente en los recién nacidos con la enfermedad en tanto que en los niños mayores, se presentan alteraciones en el cerebelo y presencia de numerosos astrocitos Alzheimer tipo II en los ganglios basales. En los neonatos se puede observar una atenuación de la sustancia blanca tanto en la tomografía como en la resonancia magnética (3).

El diagnóstico se hace con la identificación de los ácidos orgánicos en la orina: metilcitrato, propionilglicina, ácido tíglico, B hidroxipropionato, todos productos de vías alternas.

El perfil de las acilcarnitinas por espectrometría de masas en tándem, usado para la tamización, muestra carnitina disminuída y el éster de acilcarnitina (C3) aumentado. El hallazgo de acilcarnitina C4-dicarboxílica ayuda en el diagnóstico diferencial con la acidemia metilmalónica. La deficiencia de carnitina en los pacientes con acidemia propiónica se debe al aumento de la excreción renal de propionilcarnitina que comúnmente presentan estos pacientes (21). La relación propionil-carnitina/ acetil-carnitina está aumentada y la relación propionil-carnitina/palmitoil-carnitina está igualmente aumentada.

El desarrollo de la espectrometría de masas en tándem utilizando gotas de sangre en papel de filtro ha constituido un avance tecnológico muy importante en la tamización de los trastornos del metabolismo de los ácidos grasos, los ácidos orgánicos y los aminoácidos. Un estudio de Naylor y Chace en algo más de 700.000 muestras identificó 32 casos de acidurias orgánicas, incluida la acidemia propiónica, de un total de 163 errores diagnosticados $(22,23)$.

El diagnóstico definitivo se hace determinando la actividad de la propionil-CoA carboxilasa en fibroblastos o leucocitos o por la determinación de la mutación genética.

El diagnóstico prenatal puede realizarse midiendo la actividad enzimática en los cultivos de las células de la biopsia de vellosidades coriónicas o de amniocitos o por la medición de metabolitos, como el metilcitrato, en el líquido amniótico o por hallazgo de la mutación (24).

Sin tratamiento, la acidemia propiónica conlleva descompensación metabólica y encefalopatía tóxica.

El tratamiento consiste en restricción proteica $(1,5-$ $2 \mathrm{~g} / \mathrm{kg}$ por día) evitando la administración de aminoácidos precursores de propionato (valina, isoleucina, metionina y treonina), suplemento con carnitina ( $100 \mathrm{mg} / \mathrm{kg}$ por día) y biotina (10 mg por día). Se recomienda la administración de neomicina y metronidazol puesto que las bacterias del tracto gastrointestinal producen ácido propiónico. Debe administrarse un suplemento de tiamina (25) y cobalamina para evaluar la respuesta (21).

La dieta debe suministrar 120-150 cal/kg por día con un aporte de $50 \%$ de grasas y $40 \%$ de carbohidratos, y sólo el $10 \%$ de proteínas. 
Existen fórmulas comerciales especiales para el manejo de estos pacientes (26). Debe evitarse el estreñimiento, el aumento de la motilidad intestinal contribuye a la estabilización metabólica de los pacientes al disminuir las cifras de amonio sanguíneo y la excreción urinaria de propionilglicina y al aumentar la relación carnitina libre sobre carnitina total (27).

En los períodos de descompensación metabólica debe restringirse totalmente la ingestión de los aminoácidos precursores de propionato y usar la hemodiálisis o la hemofiltración si se requiere (4).

Se ha informado el trasplante hepático para el tratamiento de la enfermedad, con resolución de la anemia y mejoría significativa del crecimiento y del desarrollo intelectual (28).

La presentación neonatal se asocia a una alta mortalidad $(29,30)$. Aun en los pacientes tratados, se ha informado atrofia cerebral y aumento de lactato en el tejido cerebral (31).

En un estudio de seguimiento de seis pacientes con manifestaciones clínicas en el período neonatal, todos tuvieron algún grado de daño intelectual incluso los pacientes que no habían presentado episodios de hiperamonemia significativa. Las medidas como la gastrostomía mejoraron el crecimiento y la supervivencia (14).

La importancia de la presentación de este caso radica en que en los recién nacidos, las acidemias orgánicas y, en general, los errores innatos pueden pasar desapercibidos, confundidos con otras patologías de frecuente diagnóstico en el período neonatal como sepsis, hemorragia intracraneana, infección intrauterina, asfixia perinatal, encefalopatía hipóxica, dado que las manifestaciones clínicas de los errores innatos son inespecíficas pues el neonato posee un patrón de respuestas relativamente escaso ante las diferentes agresiones, debido a su inmadurez funcional, a la labilidad de su homeostasis y a la incapacidad para limitar cualquier tipo de respuesta a un determinado órgano (32).

\section{Informe del caso}

Se trata de un recién nacido de sexo femenino, con un peso de $3.140 \mathrm{~g}$, perímetro cefálico de 37 cm y perímetro torácico de $34 \mathrm{~cm}$. La madre tenía 40 años, G6P6A1, y el nacimiento fue por cesárea. Presentó un Apgar de 8 al minuto y de 10 a los 5 minutos.

Entre los antecedentes de importancia se encontró que padres eran primos en segundo grado, procedentes de Boyacá. La primera gestación fue un hijo vivo de 22 años con otra pareja; la segunda gestación, muerte neonatal sin diagnóstico; la tercera gestación, aborto; la cuarta gestación, gemelar, un mortinato con hidropesía fetal y un neonato con policitemia, insuficiencia respiratoria y encefalopatía que falleció a las 48 horas de vida; la quinta gestación, recién nacido a término con encefalopatía, cuadro convulsivo y apneas que ameritaron respiración mecánica asitida; durante el traslado a esta institución falleció. La escanografía cerebral simple a los cinco días de edad mostró hipoatenuación de la sustancia blanca periventricular alrededor de las astas frontales.

La recién nacida del caso que se presenta ingresó a la unidad hipotónica, hipoactiva, con succión pobre, respuesta a estímulos disminuida, sin dificultad respiratoria, con saturación normal. Presentó hipoglucemia, lactato aumentado 2,7 $\mathrm{mmol} / \mathrm{l}$, trazas de cetonuria, acidosis metabólica leve con anión gap en 21 y amonio en $182 \mu \mathrm{mol} /$ I. Se descartó bacteriemia e infección del sistema nervioso central. El ecocardiograma fue normal, al igual que la TSH neonatal.

La paciente fue remitida a nuestra institución con sospecha de enfermedad metabólica a los 11 días de vida, con peso de $3.200 \mathrm{~g}$, talla de $50 \mathrm{~cm}$, perímetro cefálico de $36 \mathrm{~cm}$, perímetro torácico de $31 \mathrm{~cm}$, con ictericia, hipotonía e hipoactividad. Se documentó hipoglucemia. En el cuadro hemático se encontró anemia, leucopenia y trombocitopenia. Se documentó infección urinaria por Klebsiella oxytoca que se trató. En el estudio metabólico se encontró cloruro férrico positivo y banda de glutamina en la cromatografía de aminoácidos en plasma y orina; el amonio fue de 63,9 micromol/l (valor normal: 9 a 33). La ecografía transfontanelar y abdominal fueron normales.

En el estudio de espectrometría de masas en tándem realizado por Pediatrix Screening se halló lo siguiente: 
Perfil de acilcarnitinas: propionil-carnitina (C3): 7,7 micromol/l; relación propionil-carnitina/acetilcarnitina $(\mathrm{C} 3 / \mathrm{C} 2)$ de 1,5 ; relación propionilcarnitina/palmitoil-carnitina (C3/C16) de 15,06; carnitina libre: $2 \mu \mathrm{mol} / \mathrm{l}$ (normal en el recién nacido: 10 a 280); acilcarnitina total, $14 \mu \mathrm{mol} / \mathrm{l}$ (normal en el recién nacido: 5 a 60); carnitina total: 16 umol/l (normal en el recién nacido: 15 a 300).

Perfil de aminoácidos: dentro de límites normales.

Con estos resultados se planteó el diagnóstico de acidemia orgánica, propiónica o metilmalónica. Con los hallazgos de carnitina libre baja, carnitina total baja, presencia de propionil-carnitina y ausencia de C4 acil-carnitina-dicarboxílica se concluyó que, posiblemente, se trataba de una acidemia propiónica.

Se inició manejo con carnitina, vitamina $B_{12}$ y dieta con restricción de proteínas. Se dio salida con igual tratamiento.

Al mes y medio de vida ingresó por un cuadro de intolerancia a la vía oral y compromiso neurológico. Se documentó acidosis metabólica con anión gap aumentado, anemia, leucopenia y trombocitopenia. Se transfundió. Hubo deterioro neurológico, el amonio estaba en 278 micromol/l. Se dejó sin aporte proteico por 48 horas. El amonio ascendió a $545 \mu \mathrm{mol} / \mathrm{l}$, se practicó transfusión extracorpórea con lo que el amonio disminuyó. Se reinició el aporte proteico, presentó cuadro convulsivo con hiponatremia, hipocalcemia, anemia, leucopenia, trombocitopenia, prolongación de los tiempos de coagulación, aumento de las aminotransferasas y compromiso multisistémico que no respondió al manejo médico y falleció al mes y 23 días de edad.

\section{Discusión}

Los antecedentes de consanguinidad, muerte neonatal sin diagnóstico, historia de hermanos previos fallecidos sin causa conocida y encefalopatía neonatal sin historia de asfixia perinatal son útiles para sospechar un error innato del metabolismo, tal como se encuentra en la historia del caso que se presenta. El hallazgo de la atenuación en las señales de la sustancia blanca en la resonancia magnética del hermano anterior fallecido permite suponer una encefalopatía por la misma enfermedad (3).

En nuestro paciente los hallazgos de anemia, leucopenia, trombocitopenia, hipoglucemia, cetonuria, acidosis metabólica con anión gap aumentado, hiperamonemia y la presencia de infección por $K$. oxytoca, un germen inusual (6), junto con los hallazgos de cloruro férrico positivo en la muestra de orina que evidencia la presencia de oxoácidos (32) y la banda de glutamina en la cromatografía de aminoácidos en capa fina en plasma y orina, presente en los pacientes que cursan con hiperamonemia, sugirieron la posibilidad de una aciduria orgánica $(1,4,11,33)$.

Se decidió practicar un perfil de aminoácidos y de acilcarnitinas por espectrometría de masas en tándem porque contábamos con el recurso y nos permitía el diagnóstico de las acidurias orgánicas y de otros errores del metabolismo $(22,23)$.

Este método es de gran utilidad en nuestro medio por las dificultades que tenemos en realizar estudios específicos y por la facilidad del manejo de la muestra al tratarse de gotas de sangre seca en una tarjeta de papel de filtro de las mismas que se usan para la tamización de hipotiroidismo congénito, única enfermedad metabólica objeto de tamización a nivel nacional.

No se realizó el estudio de ácidos orgánicos por cromatografía de gases/espectrometría de masas por falta de recursos económicos.

Recientemente, la tamización de las acidurias orgánicas usando espectrometría de masas en tandem (MS/MS) ha ganado popularidad en el mundo. La MS/MS es de bajo costo y los resultados se obtienen en corto tiempo si se compara con la GC/MS. Además, se ha descubierto un número importante de errores usando MS/MS, por lo que constituye una muy buena alternativa.

A pesar de haber logrado la compensación clínica durante la primera hospitalización, la paciente hizo una nueva crisis y falleció al mes y medio de vida, lo cual corrobora que las acidemias propiónicas de presentación neonatal son de muy mal pronóstico $(29,30)$. 
El manejo de estos niños en nuestro medio es muy difícil pues las fórmulas comerciales para la alimentación de estos pacientes y los suplementos (carnitina y biotina) no están en nuestro mercado y no se dispone de los exámenes de laboratorio necesarios para hacer un control metabólico. El diagnóstico de los casos en la época neonatal crea la necesidad de disponer de leches especiales y suplementos para su tratamiento.

El diagnóstico de esta paciente permitió hacer la asesoría genética a los padres.

Entre las causas que originan mortalidad neonatal sin diagnóstico, están los errores innatos (2), su estudio en nuestro país adquiere una importancia cada vez mayor puesto que, en la actualidad, la mortalidad neonatal causa más del $60 \%$ de la mortalidad infantil (34). De hecho, tres hermanos de la paciente habían fallecido en el período neonatal, sin diagnóstico y otro había sido una muerte fetal tardía con diagnóstico de hidropesía fetal. La consanguinidad entre los padres o la historia de abortos, mortinatos o muertes neonatales sin diagnóstico también sugieren enfermedad genética.

En el recién nacido el diagnóstico diferencial de las acidemias orgánicas en particular y de los errores innatos en general, se plantea siempre con las patologías más comunes del período neonatal: asfixia al nacer, encefalopatía hipóxicoisquémica y sepsis.

La cuantificación del amonio en todo paciente con encefalopatía es obligatoria; si se encuentra aumentado, debe investigarse una enfermedad metabólica.

En todo recién nacido que presente antecedentes familiares de consanguinidad entre los padres 0 historia de abortos, mortinatos o muertes neonatales sin diagnóstico o algún hallazgo bioclínico, que incluya encefalopatía, dismorfismo, microcefalia, sepsis que no responde al tratamiento o con hemocultivo negativo, acidosis metabólica con anión gap aumentado, hipoglucemia, cetosis o hiperamonemia se debe investigar un error innato del metabolismo. El estudio sistemático de estos pacientes nos ha permitido caracterizar la población que hemos denominado de riesgo para enfermedad metabólica (32). La realización de un perfil de laboratorio completo y la utilización de pruebas colorimétricas en orina, la cuantificación de amonio, lactato y piruvato, y la cromatografía de aminoácidos en capa fina en plasma y orina brindan un acercamiento inicial al diagnóstico de estas enfermedades $(32,35)$.

En Shanghai y Beijing se realizó un estudio de 104 pacientes de alto riesgo para enfermedad metabólica: retardo mental, hipotonía, ictericia, convulsiones, hepatoesplenomegalia, vómito recurrente, acidosis metabólica, hipoglucemia, hiperamonemia e hiperlactatemia, utilizando gotas de sangre seca en papel de filtro para espectrometría de masas en tándem. Se demostró enfermedad metabólica en 10 pacientes (36).

En nuestro medio, dadas las limitaciones de recursos económicos y tecnológicos, el desarrollo de la espectrometría de masas en tandem utilizando gotas de sangre en papel de filtro, constituye una oportunidad para el diagnóstico de acidurias orgánicas, trastornos del metabolismo de ácidos grasos y de aminoácidos, por la facilidad del envío de muestras a los centros de referencia y el corto tiempo en que se obtienen los resultados.

\section{Agradecimientos}

Al Instituto Materno-Infantil, hospital sede de la Facultad de Medicina de la Universidad Nacional de Colombia y a sus pacientes.

\section{Conflicto de intereses}

No existe conflicto de intereses y la espectrometría de masas en tandem fue realizada sin costos por la firma PEDIATRIX Screening, Bridgeville, PA, USA 15017.

\section{Financiación}

Los autores no recibieron financiación para este trabajo. La paciente estaba hospitalizada en el Instituto Materno-Infantil.

\section{Referencias}

1. Fenton WA, Gravel RA, Rosenblatt DS. Disorders of propionate and methylmalonate metabolism. En: Scriver CR, Beaudet AL, Sly WS, Valle D, editors. The metabolic and molecular bases of inherited diseases. 8 Ed. New York: Mac Graw Hill Inc; 2001. p.2165-93. 
2. Mandava P, Kent TA. Metabolic disease and stroke: propionic acidemia. [Consultado: enero 5 de 2006]. Disponible en: http://www.emedicine.com/neuro/ topic577.htm

3. Feliz B, Witt DR, Harris BT. Propionic acidemia: a neuropathology case report and review of prior cases. Arch Pathol Lab Med. 2003;127:e325-8.

4. Seashore MR. The organic acidemias: an overview. [Consultado: 3 de julio de 2007]. Disponible en: http:// www.geneclinics.org/servlet/access? $\mathrm{db}=$ geneclinics \& site $=\mathrm{gc} \& \mathrm{fc} n=y \& i d=8888891 \&$ key $=$ ZxsHOk4h-QIFE\&filename=/profiles/oa-overview/ index.html

5. Ravn K, Chloupkova M, Christensen E, Brandt NJ, Simonsen H, Kraus JP, et al. High incidence of propionic acidemia in Greenland is due to a prevalent mutation, 1540insCCC, in the gene for the beta-subunit of propionyl CoA carboxylase. Am J Hum Genet. 2000; 67:203-6.

6. Al Essa M, Rahbeeni Z, Jumaah S, Joshi S, Al Jishi E, Rashed MS, et al. Infectious complications of propionic acidemia in Saudia Arabia. Clin Genet. 1998;54:90-4.

7. Roth KS. Propionic acidemia (Propionyl CoA carboxylase deficiency). [Consultado: 24 de agosto de 2007]. Disponible en: http://www.emedicine.com/ped/topic 1906.htm

8. Sass JO, Hofmann M, Skladal D, Mayatepek E, Schwahn B, Sperl W. Propionic acidemia revisited: a workshop report. Clin Pediatr (Phila). 2004;43:837-43.

9. Boveda MD, Couce ML, Lluch MD, Merinero B, Campistol J. Protocolo de diagnóstico y tratamiento de la acidemia propiónica, metilmalónica, isovalérica. An Esp Pediatr. 1997;89:9-15.

10. Ciani F, Pasquini E, Ciardetti A, Donati MA, Zammarchi E. Hyperglycinemia in clinical-laboratory practice. Pediatr Med Chir. 1997;19:109-12.

11. Wolf B, Hsia YE, Sweetman L, Gravel R, Harris DJ, Nyhan WI. Propionic acidemia: a clinical update. J Pediatr. 1981;99:835-46.

12. Nyhan WL, Bay C, Beyer EW, Mazi M. Neurologic nonmetabolic presentation of propionic acidemia. Arch Neurol. 1999;56:1143-7

13. Filipowicz HR, Ernst SL, Ashurst CL, Pasquali M, Longo N. Metabolic changes associated with hyperammonemia in patients with propionic acidemia. Mol Gen Metab. 2006;88:123-30.

14. North KN, Korson MS, Gopal YR, Rohr FJ, Brazelton TB, Waisbren SE, et al. Neonatal-onset propionic acidemia: Neurologic and developmental profiles and implications for management. J Pediatr. 1995;126 916-22.

15. Lücke T, Pérez-Cerdá C, Baumgartner M, Fowler B, Sander S, Sasse M, et al. Propionic acidemia: unusual course with late onset and fatal outcome. Metabolism. 2004;53:809-10.
16. Mardach R, Verity MA, Cederbaum SD. Clinical, pathological, and biochemical studies in a patient with propionic acidemia and fatal cardiomyopathy. Mol Gen Metab. 2005;85:286-90.

17. Ianchulev T, Kolin T, Moseley K, Sadun A. Optic nerve atrophy in propionic acidemia. Ophthalmology. 2003;110:1850-4.

18. Barrera LA, Echeverri OY, Almaciga CJ, Malaver LF. Fundamentos de las acidemias orgánicas y desórdenes del ciclo de la urea: diagnóstico y tratamiento. Parte I. Temas Pediátricos. 2006;23:1-37.

19. Barrera LA, Echeverri OY, Almaciga CJ, Malaver LF. Fundamentos de las acidemias orgánicas y desórdenes del ciclo de la urea: diagnóstico y tratamiento. Parte II. Temas Pediátricos. 2007;24:1-25.

20. Ozand PT, Rashed M, Gascon GG, Youssef NG, Harfi H, Rahbeeni Z, et al. Unusual presentations of propionic acidemia. Brain Dev. 1994;16:46-57.

21. Pediatrix Medical Group. Propionic academia. [Consultado: 8 de agosto de 2007]. Disponible en: http:/ /www.pediatrix.com/body_screening_menu.cfm?id= 1573

22. Naylor EW, Chace DH. Automated tandem mass spectrometry for mass newborn screening for disorders in fatty acid, organic acid, and amino acid metabolism. J Child Neurol. 1999;14(Suppl.1):s4-8.

23. Chace DH, DiPerna JC, Naylor EW. Laboratory integration and utilization of tandem mass spectrometry in neonatal screening: a model for clinical mass spectrometry in the next millennium. Acta Paediatr Suppl. 1999;88:45-7.

24. Pérez-Cerdá C, Pérez B, Merinero B, Desviat LR, Rodríguez Pombo P, Ugarte M. Prenatal diagnosis of propionic acidemia. Prenat Diagn. 2004;24:962-4.

25. Matern D, Seydewitz HH, Lehnert W, Niederhoff $\mathbf{H}$, Leititis JU, Brandis M. Primary treatment of propionic acidemia complicated by acute thiamine deficiency. J Pediatr. 1996;129:758-60.

26. Yannicelli S, Phyllis B, Acosta PB, Velásquez A, Bock HG, Marriage B, et al. Improved growth and nutrition status in children with methylmalonic or propionic acidemia fed an elemental medical food. Mo Genet Metab. 2003;80:181-8.

27. Prasad C, Nurko S, Borovoy J, Korson MS. The importance of gut motility in the metabolic control of propionic acidemia. J Pediatr. 2004;144:532-5.

28. Yorifuji T, Muroi J, Uematsu A, Nakahata T, Egawa H, Tanaka K. Living-related liver transplantation for neonatal-onset propionic acidemia. J Pediatr. 2000;137:572-4.

29. Rousson R, Guibaud P. Long term outcome of organic acidurias: survey of 105 French cases (1967-1983). J Inherit Metab Dis 1984;7(Suppl.1):10-2. 
30. van der Meer SB, Poggi F, Spada M, Bonnefont JP, Ogier $\mathbf{H}$, Hubert $\mathbf{P}$, et al. Clinical outcome and long term management of seventeen patients with propionic acidaemia. Eur J Pediatr. 1996;155:205-10.

31. Chemelli AP, Schocke M, Sperl W, Trieb T, Aichner F, Felber S. Magnetic resonance spectroscopy (MRS) in five patients with treated propionic acidemia. J Magn Reson Imaging. 2000;11:596-600.

32. Cifuentes Y, Bermúdez M. Errores innatos del metabolismo en el período neonatal. En: Rojas E, Sarmiento $F$, editores. Pediatría. Diagnóstico y tratamiento. Segunda edición. Bogotá: Celsus; 2003. p.259.

33. Enns GM. Laboratorio en el diagnóstico de errores congénitos del metabolismo en el RN. NeoReviews. 2001;2:e192-200.
34. Ministerio de la Protección Social, OPS, OMS. Situación de salud en Colombia. Indicadores Básicos de Salud, 2006. [Consultado: 10 de abril de 2007]. Disponible en: http://www.minproteccionsocial.gov.co/ VBeContent/NewsDetail.asp?ID $=15895 \&$ ID Company $=3$

35. Chiaratti de Oliveira A, dos Santos AM, Martins AM, D'Almeida V. Screening for inborn errors of metabolism among newborns with metabolic disturbance and/or neurological manifestations without determined cause. Sao Paulo Med J. 2001;119:160-4.

36. Gu XF, Han LS, Gao XL, Yan YL, Ye J, Oiu WJ. A pilot study of selective screening for high risk children with inborn error of metabolism using tandem mass spectrometry in China. Zhonghua Er Ke Za Zhi. 2004; 42:401-4. 